\title{
Régulation monétaire et financière et viabilité des économies de marché
}

\section{Faruk Ülgen}

\section{(2) OpenEdition \\ 1 Journals}

Édition électronique

URL : http://journals.openedition.org/ei/465

DOI : $10.4000 /$ ei.465

ISSN : 2553-1891

\section{Éditeur}

Association Économie et Institutions

Référence électronique

Faruk Ülgen, «Régulation monétaire et financière et viabilité des économies de marché », Économie et institutions [En ligne], 17 | 2011, mis en ligne le 31 janvier 2013, consulté le 19 avril 2019. URL : http:// journals.openedition.org/ei/465; DOI : 10.4000/ei.465

Ce document a été généré automatiquement le 19 avril 2019

Revue Économie et institutions 


\title{
Régulation monétaire et financière et viabilité des économies de marché
}

\author{
Faruk Ülgen
}

Je remercie les deux rapporteurs anonymes de la revue ainsi que les Professeurs Guy Bensimon, Jaime Marques Pereira et Ramon Tortajada pour leurs remarques et suggestions qui m'ont permis d'améliorer sensiblement une version antérieure de cet article. Les erreurs et omissions relèvent, toutefois, de ma seule responsabilité.

1 La stabilité monétaire et financière fait l'objet de nombreuses recherches en économie parallèlement à l'apparition des crises d'ampleur différente dans l'évolution des économies de marché (Artus et al. (2008), Boyer et al. (2004), Capie et Wood (1986), Goodhart et Illing (2002), Honohan et Laeven (2005), Hubbard (1991), Kindleberger et Laffargue (1982), Minsky (1982, 1986), Portes et Swoboda (1987), Wolfson (1986)). Ces recherches se focalisent souvent sur une présentation typologique des difficultés survenues ou sur la classification de leurs causes particulières afin d'en déterminer les caractéristiques différenciées selon les périodes étudiées. Elles permettent ainsi d'identifier les différentes générations de crises selon les fragilités structurelles observées a posteriori dans chacune des expériences et de révéler les institutions qui en sont à l'origine (Klein et Shabbir, 2006). La question de l'évolution de la sphère financière que les déréglementations des trente dernières années auraient hypertrophiée en évinçant l'économie réelle et en détournant les capitaux vers la finance apparaît aussi comme un angle de recherche fructueux (Aglietta et Berrebi (2007), Das (2003), Mayes et Wood (2007), Sgard (2002)).

2 À l'aune des résultats bien connus de ces travaux et de la crise financière de 2007 dont les effets se sont répandus aujourd'hui à un grand nombre d'économies de par le monde, le présent article évalue la pertinence de deux principes constitutifs du mode de régulation des économies de marché à l'égard de la viabilité des relations monétaires et financières dans le temps. Le premier principe est la croyance en la capacité des marchés à corriger convenablement les erreurs de jugement ${ }^{3}$ des acteurs décentralisés. Le deuxième principe 
stipule que les schémas d'autorégulation des marchés sont plus efficaces que les formes de régulation publique.

3 Ces principes traduisent les fondements conceptuels du libéralisme économique et sont fondés sur l'hypothèse d'efficience des marchés libres. Dans une économie dominée par le principe de décentralisation des choix, fondé sur des critères et règles de décision privée, la sécurité individuelle est supposée répondre aux besoins de la stabilité systémique. En d'autres termes, la stabilité systémique, qui est de l'ordre macroéconomique, est supposée atteinte en additionnant des établissements individuels qui seraient séparément sécurisés selon certaines normes, données par exemple dans les dispositifs de Bâle I et II. Dans ce cas, il est évident que toute vision macroéconomique à long terme est subordonnée à une vision de (auto) régulation microéconomique. Or, lorsque l'on transpose l'analyse du niveau microéconomique au niveau macroéconomique, la somme des individus peut être différente du résultat macroéconomique escompté pour l'ensemble du système. Ceci fait que la stabilité ne peut pas être pensé exclusivement en termes d'éléments micro séparés. Par conséquent, lorsque les mécanismes d'ajustement sont fondés davantage sur des valeurs microéconomiques, jugées plus proches de l'éthique libérale, ils peuvent se révéler peu cohérentes pour répondre aux déséquilibres systémiques critiques. L'argumentaire qui soutient cette affirmation est présenté en deux sections.

4 La première section montre que tant l'évolution des systèmes bancaires et financiers que l'apparition de nouveaux risques sont principalement dues à la mise en place, à partir des années quatre-vingt, d'un mode de régulation qui suppose l'efficience spontanée des mécanismes de marché en réduisant la place des autorités dans les mécanismes de surveillance et d'évaluation des comportements des acteurs de marché. Toutefois, les modèles d'évaluation interne décentralisée qui découlent d'une telle orientation ne prennent pas en compte les interdépendances entre les acteurs privés et ne contiennent pas de vision macroéconomique à long terme. Ils se révèlent alors incapables de faire face à des situations de crise. De ce point de vue, l'absence de mécanismes de régulation macroéconomique apparaît comme l'une des explications de la transformation des difficultés de la période récente en une crise bancaire et financière généralisée.

5 La deuxième section considère les caractéristiques endogènes du risque systémique au travers des limites des modèles de rationalité comportementale des marchés. Une interprétation synthétique des résultats des modèles de conventions, de financement d'acquisitions par emprunt et des modèles psychologiques de dissonance cognitive est proposée. En réinterprétant le schéma d'instabilité financière de $\mathrm{H}$. Minsky à la lumière de ces résultats, on montre que la fragilisation des conditions de viabilité du système monétaire et financier est principalement due à la croyance selon laquelle les mécanismes de marché libre ont la capacité de s'autoréguler sans appeler une intervention exogène structurelle. On souligne que les marchés n'ont ni la vocation ni la capacité à assurer une régulation systémique. La viabilité monétaire et financière n'est pas du ressort des marchés décentralisés. Par conséquent, il convient d'imaginer des dispositifs macro-prudentiels en vue d'élargir le domaine de viabilité des économies monétaires et de renforcer la stabilité systémique.

\section{Efficience des marchés et nouvelle régulation}

6 Considérant l'ampleur des crises survenues dans la deuxième moitié de la décennie 1990, Rogoff pose la question de savoir s'il y a « une crise dans le capitalisme globalisé » et si 
«le système actuel est vraiment dans le besoin désespéré de réparation» (1999, p. 21). Cette question concerne le modèle libéral de fonctionnement du système économique, défini il y a plus de trente ans par Lindbeck (1979) en ces termes:

"The internationalization of credit markets and the integration of governments in those markets as regular lenders and borrowers, as well as the increased importance of market forces for exchange rate determination, mean that the coordination of government actions is increasingly brought about by the « invisible hand of markets", rather than by the more visible hand of government authorities".

7 Les fondements conceptuels de ce modèle sont fondés sur le paradigme de marchés efficients dont l'application permet une évolution spécifique des systèmes bancaires et financiers et débouche sur la primauté d'un mode de régulation particulière autorégulation - sur toute forme de coordination collective volontariste. La nouvelle régulation financière confie la coordination des activités des agents privés aux marchés déréglementés en évacuant largement les pouvoirs publics des dispositifs de régulation monétaire/financière. Remarque-t-on alors dans les orientations des autorités ou des groupes de réflexion comme le Comité de Bâle, à partir des années 1980, la prédominance des normes de sécurité individuelle consistant à demander aux établissements bancaires de procéder à l'évaluation de leur situation de risque en élaborant leurs propres modèles d'évaluation interne et en sollicitant le concours d'autres acteurs privés de marché comme les agences de notation. Sous l'effet des changements dans les mécanismes de régulation, devenus plus libéraux, les repères des actions privées sont modifiés, les acteurs se sont retrouvés en présence d'un champ d'actions extrêmement élargi et constamment réinventé. Alors qu'apparaissent de nouveaux risques dans un environnement de plus en plus autorégulé, les modèles d'évaluation interne décentralisée et le degré de crédibilité des mécanismes de réglementation rencontrent des limites sévères en période de stress et révèlent une incapacité à avoir une vision macroéconomique globale des interdépendances des acteurs sur les marchés et de leur dépendance collective vis-à-vis des modalités de résolution des crises.

\section{1. L'évolution du système bancaire et financier et les nouveaux risques}

8 Le paradigme des marchés efficients ${ }^{4}$, qui domine les conceptions contemporaines des systèmes monétaires et financiers, considère que les mécanismes de marchés libres fournissent la meilleure modalité de fonctionnement des économies tant en ce qui concerne les décisions économiques privées qu'en ce qui concerne la résolution d'éventuels dysfonctionnements, supposés dus, principalement, à des chocs exogènes. L'hypothèse de l'efficience du marché, dans la lignée des travaux d'E. Fama $(1965,1970)$, stipule que les prix de marché reflètent correctement toutes les informations nécessaires et requises ${ }^{5}$ pour que les comportements individuels séparés puissent se révéler globalement compatibles entre eux, ce qui renvoie à la définition bien connue de l'équilibre général néo-walrasien d'une économie de marché concurrentielle ${ }^{6}$. Ce paradigme affirme que les mécanismes de prix de marché reflètent à tout moment les valeurs fondamentales si bien que ni les bulles ni les krachs ne peuvent être anticipés (par définition).

Dans ce cadre, on pourrait penser, par exemple, que si des informations pertinentes sur l'évolution probable des instabilités étaient fournies par les Autorités, les agents privés 
pourraient les intégrer dans le processus de détermination des prix et prévenir toute déviation par rapport à un équilibre envisageable $\mathrm{e}^{7}$. Deux problèmes méritent alors d'être soulignés. D'une part, dans l'économie décentralisée, les Autorités ne disposent pas de cette capacité à collecter et à contrôler toutes les informations nécessaires et suffisantes sur l'évolution future des marchés, ce qui les place constamment dans une position confuse en ce qui concerne le moment opportun d'intervention contre-cyclique. D'autre part, lorsque les Autorités de régulation jugent qu'il devient nécessaire d'intervenir étant donnée l'évolution des indicateurs qu'elles estimeraient représentatifs de l'état de stabilité financière, elles devraient chercher à imposer aux acteurs des règles qui ne soient pas hypothéquées par des choix et contrôles subjectifs sur des établissements considérés séparément. Des contraintes extérieures devraient être établies afin de constituer des garde-fous susceptibles d'empêcher que les fluctuations à la hausse des prix des actifs engendrent des déviations durables jusqu'à une nouvelle crise systémique. Mais cela n'a visiblement pas été l'orientation suivie dans le passé par les Autorités qui ont opté pour des règles de régulation fondées sur la croyance des marchés efficients en abandonnant toute vision macroéconomique globale à long terme. L'option de remédier aux effets négatifs en cas de crise systémique l'emporte donc sur celle consistant à chercher à prévenir les crises d'envergure en redéfinissant les règles de fonctionnement du système financier.

10 La première option " pro-marché » choisie a marqué l'évolution des systèmes financiers depuis les années 1970 en généralisant les politiques de libéralisation et de décloisonnement des marchés ${ }^{8}$. Ce mouvement a provoqué des innovations financières élargissant et diversifiant les modalités de titrisation et de marchéisation des différents actifs et engagements au travers de multiples produits-véhicules. Les titres adossés aux actifs ont grandement contribué à l'élargissement de la capacité des banques et des institutions financières à octroyer des crédits qui ont pu aussi bénéficier de nouvelles formes de couverture. Toutefois, ces stratégies dynamiques de couverture contre les risques deviennent contreproductives dans les périodes de baisse rapide des prix en augmentant les pressions à la vente des actifs. Aussi, avec les nouveaux instruments de diversification des risques, la propagation à grande échelle d'une grande variété de risques, au fond devenus interdépendants, s'est établie en réduisant la capacité quantitative et qualitative de surveillance mais aussi d'intervention prompte des autorités. De nombreuses phases d'instabilité sont observées aussi bien sur les marchés financiers développés ${ }^{10}$ que dans les économies émergentes au travers de crises récurrentes ${ }^{11}$. Pourtant, dans son rapport sur la stabilité financière globale, le FMI (International Monetary Fund, 2003) affirmait qu'après les scandales des années 2000 dans la gouvernance des grandes sociétés, les évolutions géopolitiques instables et la bulle sur les marchés des actions, les marchés financiers avaient montré une résilience remarquable et commencé à se renforcer malgré une croissance économique terne dans les économies développées. Ce rapport soulignait plus particulièrement que les vulnérabilités sur les marchés matures avaient commencé à s'atténuer. Or, à l'observation de l'évolution depuis lors, cette amélioration se révèle toute relative et ne se fonde pas sur une solidité pérenne des marchés.

11 En effet, le développement des marchés de produits dérivés a accru la souplesse et l'ampleur des opérations de placement/financement à partir de montants de capital immobilisés de plus en plus réduits avec un effet de levier important. Si ces produits n'engendrent pas a priori sur le plan individuel d'exposition supplémentaire aux risques 
de marché12 ${ }^{12}$ ils augmentent néanmoins, sur le plan systémique, le risque de solvabilité ou de liquidité des intervenants non couverts sur le marché du risque. Par exemple, dans le cas de la titrisation adossée aux actifs (les structured investment vehicles, SIV), qui offre de nouveaux moyens de placement structurés, les opérations sont exposées à un risque de liquidité lié au décalage entre la duration de leurs passifs et de leurs actifs et au risque de crédit. Ces opérations suivent le principe de « initier puis partager » les risques et leur couverture; les banques entrent dans un engagement de financement (signifiant une prise de risque directe) et transfèrent ensuite cet engagement aux tiers en le transformant en titres négociables sur le marché financier. Par l'intermédiaire des sociétés spécifiques créées dans cet objectif ${ }^{13}$, les banques cherchent ensuite à sortir ces engagements de leur bilan. Toutefois, ce transfert de risque ne décharge nullement les banques de toute responsabilité sur la suite des évolutions puisque la crédibilité de l'émission des titres ${ }^{14}$ reste liée aux garanties de liquidité fournies par les banques sur les produits structurés dont elles ont initié le montage. Ceci est une condition de l'acceptabilité de ces produits sur les marchés. Par conséquent, en cas de retournement des marchés, les banques se retrouvent, au fur et à mesure que le stress gagne en ampleur, au premier rang face à la demande de conversion des titres en monnaie. La souplesse requiert en même temps davantage d'engagements "dissimulés", exposés indirectement aux risques globaux. Et l'apparente désintermédiation des marchés renforce au contraire l'implication des banques dans les opérations de financement de l'économie tant du côté des investissements productifs qu'en ce qui concerne les crédits à la consommation des biens durables et non durables. Mais, face à de tels retournements, il n'existe plus de mécanisme d'intervention exogène préventive capable de réorienter les comportements des acteurs, les mécanismes de réglementation macroéconomique étant remplacés par une autorégulation.

\subsection{La nouvelle réglementation : les impasses de l'autorégulation}

Les mécanismes de réglementation bancaire et financière, modifiés radicalement les trente dernières années, confient l'ajustement des déséquilibres aux modèles de rationalité microéconomique. Le besoin de régulation globale est supposé satisfait par des procédures décentralisées d'autorégulation qui devraient être établies sur des mécanismes d'incitation microéconomique, appuyés par des infrastructures juridiques et techniques "pro-marché ». Au travers d'une comparaison de plusieurs expériences de crises dans de nombreux pays, Barth, Caprio et Levine (2006) soutiennent que les réglementations restrictives sur les activités bancaires se révèlent moins efficaces que les formes plus libérales. Dans cette lignée, le retrait régulier des Autorités au profit du principe d'autorégulation des marchés correspond à un nouveau modèle, appelé par Mishkin (2001) « l'approche de la supervision ». Par opposition à l'approche prudentielle contraignante, ce modèle prône pour un contrôle, par évaluation ex post, de la qualité de gestion des activités bancaires et suppose que les agents privés ont la capacité à gérer et à contrôler eux-mêmes les risques qu'ils prennent sur les marchés. On remarque, par exemple, qu'après le guide proposé en 1993 par la Federal Reserve sur l'examen des activités de trading et de marché des dérivés, la Federal Reserve et l'office of the Comptroller of the Currency (OCC) ont annoncé en 1995 une nouvelle modalité de contrôle de la gestion des risques bancaires proposant un système de notation allant de 1 à 5 dans le cadre du système CAMELS. 
13 les gouverneurs des banques centrales du G10, au lendemain de l'abandon du régime de changes fixes de Bretton Woods, constitue un exemple probant à l'application politique du nouveau modèle. Le Comité avait proposé dans un premier temps (accords de Bâle I de 1988) un dispositif, résumé dans le ratio Cooke, qui a rapidement rencontré ses limites dans la réglementation des activités de marché des établissements bancaires. Sous l'effet des opérations d'arbitrage prudentiel effectuées par les banques pour échapper aux contraintes sur leurs engagements de crédits et avec la croissance des dérivés et des risques hors bilan subséquents, le Comité a redéfini de nouvelles normes réglementaires (accords de Bâle II), cristallisées dans le ratio McDonough en 2004. Ce travail, étalé sur 1998-2005, traduit la nouvelle orientation réglementaire en fondant la définition d'une mesure plus pertinente du risque de crédit principalement sur un système de notation interne à chaque établissement concerné. Ainsi, le Comité a proposé en juin 1999 que les banques utilisent davantage leurs modèles de risque de crédit pour établir des ratios prudentiels appropriés. À partir de 2004, les recommandations soutiennent la mise en place d'un système d'évaluation composé d'une méthode dite standard qui consiste à utiliser des systèmes de notation proposés par des agences de notation et d'une méthode de notation interne propre à chaque établissement (Internal Rating Based, IRB) ${ }^{15}$. Ainsi, l'évaluation de la gestion des fonds est principalement confiée à des procédures internes de surveillance (IRB), accompagnées des procédures de conseil et de notation par des agences privées qui, bien qu'en apparence extérieures aux établissements évalués, sont des pratiques qui demeurent internes aux mécanismes de marché. La discipline du marché, qui devrait en garantir la cohérence, est supposée satisfaite par la transparence dans la communication des établissements. Dans ce cadre, les banques fixent elles-mêmes les pondérations des risques. Malgré la critique que l'on a adressée au ratio McDonough d'être excessivement rigide ${ }^{16}$, il y a une plus grande souplesse pour les banques dans l'évaluation de leurs risques du fait de l'élargissement de l'éventail des pondérations. En effet, les trois piliers sur lesquels la nouvelle réglementation ${ }^{17}$ s'appuie sont explicitement montés sur le principe d'autorégulation. Le pilier I, qui porte sur l'exigence de fonds propres en cohérence avec les risques encourus par les établissements, laisse les établissements libres d'utiliser les méthodes standard et d'IRB globalement subjectifs. Le pilier II, qui détermine la procédure de surveillance de la gestion des fonds propres, met en avant la transparence et la responsabilité prudentielle des établissements en leur demandant de faire valider ex post la pertinence des méthodes et des données utilisées dans leur calcul ${ }^{18}$. Le pilier III est appelé la discipline du marché à travers l'uniformisation de « bonnes pratiques » en matière des données et informations. augmenté leur activité de titrisation et accru leur prise de risque, accompagnées d'une forte hausse des retours sur investissement, comme le souligne le Rapport Larosière de février 2009. Ceci a engendré le phénomène de shadow banking enlevant toute efficacité aux mécanismes de surveillance prévus. Deuxièmement, l'importance des agences de notation dans l'évaluation de la situation des établissements est devenue déterminante. Ce rôle assigné aux agences a permis la coexistence de deux activités contradictoires en leur sein; activité de conseil et activité d'évaluation. En effet, particulièrement dans l'évaluation des crédits structurés ${ }^{19}$, l'élaboration des différentes catégories de crédit et leur évaluation sont fondées sur l'intervention permanente des agences en tant que conseillers techniques des banques. Cela engendre une confusion de fait entre le métier 
de conseil et celui d'évaluateur, la fonction de perte du dernier suivant inéluctablement la fonction d'utilité du premier. En d'autres termes, la tâche d'évaluation et plus particulièrement les informations qui en découlent, qui peuvent être considérées comme des biens publics ${ }^{20}$, deviennent dépendantes d'une activité privée, le conseil. Se crée alors ouvertement un conflit d'intérêt puisque les agences sont à la fois conseiller des établissements dans les montages des différents produits financiers et évaluateur des mêmes produits. On comprend alors qu'elles ont tout intérêt à ce que les mécanismes de régulation soient de plus en plus dépendants de leurs notations; les établissements et produits à évaluer constituant leur principal marché21. On pensait que ces sociétés d'audit et de conseil, de réputation mondiale et évoluant sur un marché stable ${ }^{22}$, suivraient une autodiscipline selon des mécanismes d'incitation des marchés concurrentiels, contrairement aux insuffisances supposées des formes de contrôle centralisées et bureaucratiques, soulignées entre autres par Lorenzi (2008). Les règles d'évaluation suivant les seules valeurs de marché ont rendu toutefois les activités de notation hautement déstabilisantes pour les sociétés cotées. Securities and Exchange Commission Américaine avait déjà questionné les risques inhérents aux mécanismes en vigueur suite aux scandales d'Enron et de WorldCom (SEC, 2003). Cette commission avait souligné les conflits d'intérêt et la nature exclusivement micro-prudentielle des évaluations appliquées. Dans cette perspective, Sy (2009) remarque aussi le caractère pro-cyclique des activités de notation qui exacerbent souvent les comportements grégaires sur les marchés avant de procéder à des dégradations brutales en période de crise $^{23}$.

D'une façon plus générale, il s'avère que ni les modèles internes des établissements bancaires ni les agences de notation ne permettent de détecter de graves anomalies jusqu'au début des crises. Comme les différentes catégories de risque et d'agents sont supposées indépendantes dans les modèles d'évaluation, l'estimation d'un risque global ne fait en général pas l'objet d'une étude systématique et suivie « (...) principalement à cause de la dissonance cognitive qui rend très difficile d'avoir une attitude de doute à l'égard de ses propres croyances » (Aglietta, 1998, p. 59). Les établissements de crédit utilisent le modèle de Value-at-Risk (VAR) qui suppose les chocs comme stationnaires et les interactions entre les opérateurs stables dans le temps. Aussi, la couverture du risque d'un portefeuille donné implique que le risque soit correctement évalué à travers les primes de risque appliquées. L'évaluation des engagements des établissements est fondée alors sur l'hypothèse d'absence de perturbations susceptibles d'émerger des interactions entre les engagements des agents sur les marchés. Toutefois, l'hypothèse de l'exogénéité des chocs sur le plan individuel se révèle erronée en période d'instabilité macroéconomique. Car lorsqu'un nombre significatif d'agents cherchent à liquider rapidement les positions cumulées, une panique généralisée envahit les marchés et toute anticipation de reprise des opérations habituelles s'évanouit.

Les acteurs privés sont dans l'incapacité de considérer leurs interdépendances mutuelles dans leurs évaluations internes puisque les interdépendances sont de nature macroéconomique alors que les systèmes d'évaluation individuelle (autorégulation) ne comprennent pas, par définition, de mécanisme de régulation macroéconomique systémique. La capacité du marché à assurer la coordination nécessaire entre les décisions individuelles en cas de stress s'en trouve d'autant plus réduite. L'absence de vision macroéconomique de moyen et long termes détermine la faiblesse des mécanismes de coordination spontanée qui se révèlent insuffisamment (positivement) réactives face à des situations de stress (Aglietta, 2003). Une des conséquences remarquables de la 
supervision libérale et décentralisée est alors de réduire le champ d'action des Autorités et de limiter la portée systémique de leur intervention en cas de crise d'envergure. L'aptitude et la disposition des " pompiers » ne paraissent pas suffisamment robustes à l'égard de l'incendie étendu, ce qui empêche les marchés d'évaluer rapidement la portée positive des actions autoritaires et de recouvrer leur confiance.

Dans les modèles classiques (Bryant, 1980, Diamond et Dybvig, 1983), les paniques bancaires peuvent être auto-réalisatrices étant données la vulnérabilité des engagements des banques aux paniques soudaines, mais aussi leur rôle nodal dans le processus de financement des économies. Ceci a été maintes fois observé dans les crises qui ont secoué les économies émergentes ces trente dernières années. Cette observation se renouvelle aussi dans la crise actuelle partie, cette fois-ci, d'un marché central développé, celui des États-Unis. Or, d'importants travaux ont été effectués dans les années 1990 à la recherche de mécanismes optimaux de surveillance et d'intervention qui affirment l'optimalité d'une régulation mixte au travers des systèmes de garantie partielle des créanciers (Bhattacharya, Boot et Thaker, 1998). La régulation mixte, fondée à la fois sur un mécanisme public de sécurité et de surveillance sur les opérations de marché et sur le contrôle et la discipline du marché, serait plus à même de répondre aux déséquilibres provoqués par les fragilités des relations d'endettement et au problème d'aléa moral susceptible de provenir des comportements des agents preneurs de risque. Toutefois, ces modèles, d'obédience micro-prudentielle, sont exclusivement fondés sur deux hypothèses restrictives: l'exogénéité des chocs (de liquidité et de solvabilité) et l'absence d'interdépendance entre les créanciers et les débiteurs sur différents marchés.

L'intervention des Autorités est susceptible de calmer les incertitudes des marchés si les agents font confiance en la capacité des autorités à contrecarrer les effets des éléments de détresse. Mais étant donnée la faiblesse des mécanismes de régulation macroéconomiquesystémique, cette capacité semble excessivement réduite ${ }^{24}$. Tant que les croyances opportunistes sont reconduites sur les possibilités de réaliser de gains substantiels sur des marchés libéralisés, les agents ne questionnent évidemment pas la capacité d'intervention des autorités en cas de panique ni l'évolution probable du système de paiements dans le futur. Comme Akerlof et Shiller (2009, pp.54-55 et 119) le soulignent, avant la crise financière de 2007, les prêts hypothécaires, y comprises les tranches les plus risquées (subprimes), étaient notés dans leur grande majorité en haut de l'échelle obtenant des notes AAA si bien que juste avant le déclenchement de la crise, les primes de risque étaient à leur plus bas niveau. En toute logique, l'interrogation sur la stabilité macroéconomique revient aux Autorités publiques qui n'ont pas d'intérêt de gain dans le jeu des marchés. Tout équilibre qui dépend seulement des anticipations sur la continuité des opportunités est fragile et vulnérable aux retournements soudains sur les marchés. Lorsque de tels retournements deviennent réels, les agents n'ont plus le luxe de croire raisonnablement à la possibilité d'une "reviabilisation" des rapports antérieurs par l'intervention des autorités. Il devient inimaginable que les anticipations des agents puissent s'agréger à aucun avenir joyeux. Les sommes publiques colossales injectées sur les marchés depuis 2008 ne permettent plus alors de créer cette croyance nécessaire en un retour rapide au calme puisque les mécanismes de régulation en vigueur ne se révèlent pas suffisamment puissants et efficaces face à une crise persistante.

19 Le pêché originel du mode de régulation contemporain est lié à la confusion entre l'économie de marché (et sa stabilité) et la déréglementation monétaire et financière 'exubérante'. En effet, il semble que les mécanismes d'autorégulation omettent le 
nécessaire questionnement sur les conditions de viabilité globale des systèmes monétaires, liées à l'existence d'ancrages des mécanismes faisant inéluctablement appel à des règles collectives supra-individuelles. De ce point de vue, les difficultés observées depuis le déclenchement de la crise en 2007 devraient être pensées non pas en termes standard de l'opposition « plus d'État ou moins d'État » mais à travers une interrogation sur les possibilités d'une continuité des relations économiques lorsque la logique microéconomique et les exigences macroéconomiques ne sont pas trivialement compatibles. Cette incompatibilité devient visible dans les dysfonctionnements de la nouvelle régulation.

\section{La stabilité et la viabilité systémique dans l'économie monétaire}

20 En raison de la grande diversité des variables qui peuvent affecter la stabilité des systèmes monétaires/financiers et intervenir dans l'occurrence et l'évolution des crises, il n'existe pas de modèle unifié de crises financières. Le travail colossal de C. Kindleberger (1989) témoigne de la complexité des crises dans le passé. Toutefois, il semble exister aujourd'hui une vue consensuelle selon laquelle les économies et les intervenants sont en mesure d'accroître la qualité de leurs décisions au travers d'un processus d'apprentissage à partir de leurs erreurs passées. Ceci rendrait plus solides et plus adéquates les politiques macroéconomiques et les stratégies financières des agents privés sur les marchés. La probabilité d'apparition des crises systémiques en serait alors d'autant plus réduite en circonscrivant les épisodes de perturbation à des marchés peu développés et incomplètement libéralisés. Or, comme Reinhart et Rogoff le soulignent (2008), une telle vision est peu fondée dans la mesure où, malgré les changements technologiques et économiques, différentes instabilités se suivent depuis, au moins, deux siècles et les périodes d'euphories qui se terminent «en larmes» semblent constituer une constante dans l'évolution de nos économies. L'appréhension de cette constance peut être fournie par l'analyse du caractère endogène de l'instabilité systémique et de la fragilité des conditions de viabilité des systèmes monétaires et financiers.

\section{1. Risque de système et confusion dans les comportements microéconomiques}

Lorsqu'un ou plusieurs événements perturbateurs, a priori à caractère local et/ou individuel, se produisent et poussent le système monétaire et financier vers une zone critique d'instabilité telle que le contrôle de l'évolution macroéconomique devient problématique et les difficultés rencontrées commencent à se propager à l'ensemble de l'économie, le risque de système apparait. Ici, nous proposons une lecture du risque systémique différente des modèles standard de crise financière qui sont en général fondés sur une description ex post des circonstances dans lesquelles les instabilités surviennent. Cette lecture alternative s'appuie sur deux observations qui caractérisent le fonctionnement des économies financiarisées.

D'une part, l'environnement financier très libéralisé permet de nombreuses innovations bancaires qui se développent à l'intérieur des structures monétaires/financières en réseau et qui créent de fortes interdépendances entre différents acteurs (individus, 
groupes, institutions ou pays). Par conséquent, les comportements microéconomiques (décisions et actions unilatérales des acteurs) deviennent susceptibles de générer des effets multilatéraux et multi-niveaux tels qu'en période de perturbations non contrôlables, des réactions en chaîne sur de nombreux marchés se produisent rapidement, indépendamment des anticipations antérieures des agents sur l'évolution future de l'économie. Les perturbations non contrôlées et étendues peuvent provenir aussi bien des chocs externes qu'internes à une économie donnée.

D'autre part, sur le plan fondamental, les instabilités qui sont liées à ces perturbations se révèlent souvent endogènes aux marchés. Le caractère génétiquement incomplet/ imparfait des systèmes monétaires/financiers dans les économies décentralisées rend le potentiel d'instabilité une caractéristique propre permanente. Dans cette perspective, une référence pertinente est l'hypothèse d'instabilité financière de H. Minsky $(1982,1986)$ qui suppose que les instabilités financières sont préparées durant les périodes d'euphorie généralisée sur les marchés. Or, il est possible de développer ce schéma Minskien en prenant en compte certains éléments particuliers de la dynamique d'alimentation des phases d'euphorie. Ces éléments sont à trouver dans les résultats des modèles de conventions (Orléan, 2004, Bourghelle et al., 2005), des modèles de financement d'acquisitions par emprunt qui s'appuie sur un effet de levier bancaire, LBO (Kaplan et Stein, 1993), et des modèles psychologiques de dissonance cognitive (Festinger, 1957, Akerlof, 2005). Une réinterprétation des résultats de ces travaux permet de considérer la préparation, l'apparition et l'alimentation des crises monétaires et financières (quelles qu'en soient les circonstances particulières) dans un schéma de cycle financier endogène, pour reprendre la présentation de M. Aglietta (2003).

24 En effet, une première phase, qui peut être identifiée comme la phase de préparation de la crise, révèle une évaluation très positive des actifs par les agents. Elle est fondée sur une convention implicite qui dicte les décisions des acteurs: tant que les marchés semblent considérer que le potentiel de valorisation des activités économiques n'est pas encore atteint, les anticipations privées sur leur devenir restent optimistes et la demande continue d'alimenter les prises de position à la hausse. Par conséquent, les entreprises continuent leurs stratégies d'endettement et de rachat au travers des LBO alors que les banques et intermédiaires financiers nourrissent la production et la circulation des produits très souples et peu gourmands en capital (comme les dérivés, options et futures) pour permettre le financement continu de telles opérations. Toutes les parties engagées restent confiantes anticipant de nouvelles opérations plus rentables avec un appétit à toute épreuve. Car même lorsque certains indicateurs d'alerte peuvent se situer dans des zones critiques, les intervenants continuent de penser qu'une éventuelle crise ne se produira que "plus tard» et qu'il est préférable de multiplier les opérations à rendements élevés pour ne pas laisser passer des occasions offertes par les croyances positives des marchés sur l'évolution des économies.

De ce point de vue, l'interprétation proposée dans cet article réfute l'idée répandue que les instabilités sont préparées par les comportements des intervenants malhonnêtes qui chercheraient à léser les marchés afin d'obtenir des gains personnels indûment élevés sans penser aux conséquences macroéconomiques de leur agissement sur les individus plus honnêtes. Dans un environnement euphorique, les intervenants croient en leur propre anticipation chimérique. L'explication de cette affirmation est fondée sur la deuxième caractéristique de la dissonance identifiée par Akerlof (2005) qui stipule que les individus ont tendance à manipuler leur propre croyance en sélectionnant les 
informations de façon à ce qu'elles confirment leur croyance désirée (ils «s'automentent ", diraient les psychologues). Il convient de remarquer aussi que les acteurs privés ne sont pas en mesure de savoir, par la définition même d'un programme d'optimisation microéconomique, quand ils devraient arrêter, individuellement, leur engagement et se retirer d'un marché qu'ils supposent encore porteur d'opportunités de gain - ne serait-ce que pour un très court instant supplémentaire -. Ceci est une hypothèse tout à fait logique puisque les acteurs ne disposent pas d'une information précise sur ce que serait l'évolution future de l'économie s'ils continuent d'agir ainsi ; une telle information dépasse leur capacité micro-individuelle ${ }^{25}$. L'objectif de réaliser des gains lorsque l'on pense qu'il existe encore des opportunités inexploitées ${ }^{26}$ amène les agents à entrer dans des positions au-delà de ce qui se révélera ex post être leur capacité de risque. C'est pourquoi, les modèles d'évaluation des risques restent, d'une part, au niveau micro-individuel et sont fortement entachés de la subjectivité des anticipations relatives à chaque intervenant et, d'autre part, très imprégnés du problème de la dissonance cognitive qui domine les comportements des agents dans un monde décentralisé et incertain.

Dans la deuxième phase, certains chocs, dus, par exemple, à des difficultés dans les positions bilancielles de quelques établissements de crédit, se révèlent critiques lorsqu'ils commencent à provoquer des externalités négatives sur d'autres acteurs. Les anticipations optimistes cèdent devant l'accroissement de l'incertitude concernant les possibilités de reconduction des relations de marché. Des incidences macroéconomiques qui apparaissent alors renforcent une méfiance qui se généralise alors que quelques instants plus tôt tous les établissements se situaient encore dans un optimisme de Panurge mais aussi pantagruélique. Lorsqu'il n'existe pas de mécanisme avéré d'autocoordination et d'auto-ajustement en détresse, la contagion de la méfiance l'emporte et les risques ressentis par les agents ${ }^{27}$ paraissent trop lourds à porter pour pouvoir continuer les opérations avec le même optimisme qu'auparavant. La viabilité des opérations de réseaux interbancaires est alors mise en doute. Malgré la disponibilité d'importantes liquidités, le marché monétaire est asséché, les opérations de refinancement interbancaires étant suspendues. Les positions ouvertes accumulées deviennent insoutenables sous le poids de la perception des fragilités individuelles, ce qui joue en amont en faveur du déclenchement de la panique.

Dès lors, les trois déterminants des comportements (convention, évaluation par LBO et dissonance cognitive) se révèlent confus :

- La convention sur la valorisation des actifs n'apparaît plus crédible avec l'éclatement de la bulle. On ne considère plus que les prix des actifs continueront d'augmenter et que les débiteurs de contracter de nouveaux crédits en contre partie de leurs futures richesses subséquentes ;

- Le levier d'endettement s'avère non tenable faute de financement (assèchement de liquidité ou d'opportunités). Il n'apparaît plus possible de continuer à alimenter la dette des agents dont les activités ne semblent plus en mesure de fournir des rendements de plus en plus élevés. Au contraire, le niveau cumulé des dettes se révèlent excessifs vis-à-vis de la capacité de remboursement des débiteurs, mais aussi à l'égard du niveau atteint de leur richesse ;

- La croyance autoalimentée par la dissonance cognitive revient à une « réalité » différente de la veille (accroissement de l'incertitude systémique). Personne n'est plus en mesure d'affirmer que la croissance spéculative pourra continuer encore longtemps en fournissant 
ainsi des gains supplémentaires capables de justifier le bien-fondé (la rentabilité) des positions financières accumulées.

Le problème de ce « retour à la réalité » est que, au lieu de se présenter comme un simple (et naturel) processus d'ajustement (ou d'homéostasie), le processus de "correction des exubérances" s'apparente souvent à une crise douloureuse et couteuse en termes économiques et sociaux qui ne permet pas d'assainir les structures défaillantes antérieures. Car la perception des difficultés de quelques établissements se transforme aux yeux de tout le monde en le dysfonctionnement de toute la structure relationnelle en remettant en cause la reproduction du système de valeurs financières. La panique se conclut par le repli de tous les agents, ceux-ci se plaçant du même côté du marché et s'interdisant du même coup toute possibilité de couvrir leurs positions puisqu'ils constatent qu'il n'y aura pas de contrepartie en face des ventes éventuelles. Sur les marchés spéculatifs, les engagements commencent à se réduire fortement et les positions sur des arbitrages non couverts sont précipitamment dénoncées. Le seul moyen pour continuer une existence économique sociale reste l'intervention des pouvoirs publics.

On voit aujourd'hui que la réallocation massive des portefeuilles n'est pas réalisable même lorsque les marchés financiers sont très développés (profonds, larges et liquides). Certes, en temps normal, la propagation des perturbations devient plus diffuse grâce au nombre élevé de marchés et à l'existence de nombreux intermédiaires. Mais cela ne permet pas d'augmenter la résilience des marchés développés aux crises systémiques au travers de micro ajustements. L'incertitude dominante ne peut être contrecarrée ni par la taille du marché et le nombre de produits diversifiés ni par les capitaux liquides disponibles sur l'ensemble des marchés. Cette situation critique ne peut être dissipée que par la croyance (la confiance) des agents privés qu'il existe des mécanismes collectifs adéquats et pertinents pour faire face, structurellement et systématiquement, aux errements des stratégies individuelles de 'sauve-qui-peut'. Il en apparaît que la viabilité $\mathrm{du}$ système dépend des conditions qui ne peuvent pas être remplies par les seuls mécanismes de marché. L'impossibilité d'une réaction structurée et volontariste spontanée et auto-organisée de la part des marchés en cas d'instabilité fait appel à des systèmes publics de régulation.

\section{2. La viabilité et les fausses croyances}

30 En termes dynamiques, la viabilité d'un système dans le temps est liée à la pertinence des mécanismes de reproduction et d'extension en vigueur et à sa capacité de répondre aux éventuels dysfonctionnements internes et aux chocs externes sans remettre en cause ses principes fondamentaux d'existence. Or la crise actuelle semble remettre en question certains des principes fondamentaux du mode de régulation du système économique libéral, en vigueur depuis les années quatre-vingt dans une grande partie des économies du monde. Ces principes se révèlent incapables d'assurer la continuité des rapports économiques sous leur forme actuelle (Ülgen, 2011). Un premier principe défectueux est fondé sur la croyance en la capacité des marchés à corriger d'une façon «douce » les erreurs de jugement des acteurs décentralisés. Le deuxième principe stipule que les mécanismes de régulation par les marchés (autorégulation des acteurs privés et des marchés décentralisés) sont plus efficaces que les mécanismes de régulation publique.

31 La croyance en la capacité des mécanismes de marché libre à corriger convenablement les éventuels déséquilibres est fondée sur l'idée que dans un monde libéralisé, les petites 
crises (krachs boursier, immobilier, crises de change, instabilités monétaires et bancaires récurrentes) constitueraient un moyen efficace de répondre régulièrement aux problèmes accumulés des dysfonctionnements des marchés sans nécessiter une remise en cause du libéralisme ni une intervention structurelle des pouvoirs publics. Ces pseudocrises ou perturbations financières sans panique bancaire généralisée seraient alors la preuve que le système financier est "capable de sécréter lui-même les antidotes aux crises et bien loin d'être fragilisé au fur et à mesure qu'il devient plus complexe, il en viendrait même à réagir plus vite, peut-être même plus efficacement, que le classique prêteur en dernier ressort » (Lacoue-Labarthe, 1992, pp. 409-410). Toutefois, les crises successives ne semblent pas être des moyens adéquats de réduire les pressions des dysfonctionnements des marchés sur l'économie, mais au contraire des indications traduisant les difficultés de fonctionnement stable à moyen et long termes des marchés déréglementés. Les interventions massives, souvent qualifiées de «non conventionnelles ", des autorités monétaires et la recherche de nouvelles modalités de réglementation financière plus contraignantes, même sous des formes relativement réservées et passives des instances internationales de décision comme le G20, montrent que seule la main visible des mécanismes d'action collective est en mesure d'ériger des barrières devant les exubérances décentralisées. Ainsi, Kindleberger soulignait-il en 1997 que bien qu'il nous soit difficile, voire impossible de prévoir si des difficultés financières vont ou non se conclure en une crise majeure, le principal enseignement que l'on peut tirer (ou que nous aurions dû tirer) des crises passées est la fonction essentielle du prêteur en dernier ressort. Etudiant les crises et paniques survenues aux États-Unis avant l'établissement de la Federal Reserve, Akerlof et Shiller (2009, pp. 83 et 111) soulignent que prévenir une énième panique en permettant de rétablir la confiance a été la raison fondatrice de la banque centrale américaine.

Lorsque l'on considère les trois dernières décennies, on observe un enchaînement de difficultés successives sans relâche dans des économies ou régions très différentes. Pour ne reprendre que la dernière décennie, P. Artus (2007, p. 225) remarque un schéma récurrent du type : montée d'une bulle (prix d'actifs et endettement) $\rightarrow$ explosion de la bulle $\rightarrow$ démarrage de la bulle suivante, ce qui signifie que l'évolution «normale » de l'économie est constituée d'une succession de crises entrecoupées de périodes d'euphorie (de bulle). Ainsi, la deuxième moitié des années 1990 correspond à l'euphorie des valeurs technologiques et des acquisitions spectaculaires dont la bulle éclate au début de la décennie suivante provoquant le désendettement des entreprises. Le remplacement de la cible d'euphorie s'effectue à partir de 2002-2003 par le gonflement de la bulle immobilière partout dans le monde alors qu'une bulle de ce type avait déjà posé des problèmes lors de la crise asiatique de 1997. Shiller (2005) montre, dans une perspective historique, que lorsque les profits recommencent à croître fortement, l'enthousiasme d'une nouvelle période de croissance gagne de l'ampleur en entraînant à nouveau les acteurs dans une course folle. Les investisseurs croient alors qu'ils sont sur une bonne piste dont le bienfondé n'est plus à questionner, ce qui confirme l'interprétation de la dynamique des comportements des marchés proposée au paragraphe précédent. C'est probablement dans ce sens que l'euphorie immobilière de 1995-2005 a pu se développer avant d'atteindre ses limites à partir de fin 2006 et de devenir l'élément déclencheur de la crise actuelle. En même temps, une nouvelle bulle, celle des prix de certaines matières premières, se forme en anticipant le retournement de la bulle immobilière, ce qui semble s'arrêter aujourd'hui, et ce d'une façon très confuse, par la généralisation et l'aggravation de la crise. On voit donc la formation, tous les 4 à 5 ans d'une bulle spécifique, cherchant à 
canaliser les fonds vers de nouvelles opportunités. Toutefois, cette réallocation n'assainit pas structurellement les marchés en les réorientant vers un régime d'accumulation qui serait fondé sur une croissance productive qui améliorerait, entre autres, la répartition des revenus en créant les moyens d'une reprise par une demande financièrement soutenable.

De ce point de vue, il conviendrait de remarquer qu'une relecture du schéma Minskien d'instabilité financière est nécessaire. En effet, la présentation habituelle de l'hypothèse d'instabilité financière endogène renvoie à des périodes de croissance "classique ${ }^{28}$, suivies des périodes de récession plus ou moins critique. Or, ce que l'on observe dans l'ère de libéralisation financière des trente dernières années correspond plutôt à des périodes d'euphorie spéculative, très peu ou pas du tout fondées sur une croissance classique, mais plutôt sur une croissance par le gonflement de la dette sans que cette dernière soit accompagnée par une amélioration du niveau des revenus, capable de soutenir une demande durable. Les crises qui s'ensuivent se présentent alors comme des crises d'appauvrissement généralisé en détériorant davantage la soutenabilité d'une demande prochaine et en préparant le terrain à une nouvelle période d'euphorie spéculative très court-termiste.

nouvelle donne actuelle ne paraît dès lors pas en mesure d'empêcher l'apparition de crises d'envergure ni dans les économies émergentes, qui subissent les contrecoups des déséquilibres sous forme de "surchauffe » économique difficilement contrôlable (comme en Chine et en Turquie) ou de déséquilibres des balances de paiements dépendant des fluctuations de la demande mondiale et des prix des matières premières (comme en Argentine ou en Turquie); ni dans les pays avec des marchés financiers développés, qui cherchent à atténuer les effets des déséquilibres en appliquant des politiques fiscales et des revenus récessives (comme dans l'Union européenne).

Le deuxième principe, qui suppose l'efficacité de l'autorégulation par rapport aux formes de régulation publique, est fondé sur l'idée qu'en présence d'institutions capables de soutenir les mécanismes de marchée ${ }^{29}$, les schémas d'autorégulation permettraient d'augmenter la résilience des économies aux divers chocs et de pérenniser la stabilité du fonctionnement libre des marchés (Ülgen, 2011). Or, la crise actuelle, en gestation depuis plusieurs années et très remarquable dans son étendue et son ampleur, rappelle un principe universel: il n'est pas possible d'être à la fois juge et partie lorsqu'il faut surveiller, évaluer et, le cas échéant, sanctionner des activités privées intéressées. Sy (2009) et Cantor et Mann (2009) montrent que le principe d'autorégulation à travers les agences privées impliquées dans les activités de marché des banques génère un mouvement pro-cyclique en alimentant la croissance financière dans les périodes de boom et en arrêtant abruptement l'évolution des prix des actifs dans les périodes de détresse au lieu de jouer un rôle stabilisateur contre le gonflement des bulles systémiques.

Peut-on affirmer que les établissements bancaires et financiers sont parvenus aujourd'hui à réduire considérablement le risque élémentaire (risque de liquidité et de solvabilité des intervenants non couverts sur le marché) et le risque de liquidité global grâce au développement de la surveillance interne des risques de marché ? Ce n'est pas parce que les problèmes rencontrés sur les marchés centraux (des pays développés) depuis quelques décennies ne se sont pas transformés à chaque fois en une crise de système que l'on pourrait penser que les agents du marché sont devenus capables de s'autoréguler. Même sans prendre en compte l'expérience de la crise de 2007, il apparaît clairement, à qui sait 
considérer le problème avec tant soit peu de cohérence logique, que rien ne peut valider l'affirmation selon laquelle les établissements engagés dans les opérations de marché peuvent devenir raisonnablement à la fois évaluateurs et évalués, surveillants et surveillés. Ceci est d'autant plus vrai qu'une partie considérable de ces établissements sont les banques, c'est-à-dire les institutions monétaires qui sont directement liées à l'existence du système de paiements. Or, avec l'évolution des marchés déréglementés, les banques et les intermédiaires financiers non monétaires (par exemple, les sociétés d'assurance) sont en concurrence directe sur les marchés de placements mobiliers et immobiliers et sont à l'affût de la moindre opportunité financière à court terme sans considérer spontanément et d'une façon autorégulée l'évolution de leurs positions courtes et spéculatives. Il n'est pas possible de croire que les acteurs du marché internaliseront les risques systémiques de façon à prévenir ou à corriger les comportements déstabilisants sur les marchés. Car, il paraît logiquement inévitable que les décideurs des grands établissements de marché s'engagent dans des opérations si besoin est de plus en plus risquées et complexes, dans l'objectif de ne pas laisser passer les opportunités de profits supplémentaires tant qu'ils croient que le système le permet. Ce comportement se situe, contrairement à l'interprétation qui en est donnée par Akerlof et Shiller (2009), tout à fait dans la droite ligne du concept de rationalité microéconomique. Comme souligné plus haut, les agents privés décentralisés ont procédé à des stratégies individuellement rationnelles sans être inquiétés par les éventuelles conséquences macroéconomiques de leurs actions, ce qui n'était de toute façon ni de leur portée ni dans leur programme d'optimisation.

Toutefois, malgré la persistance des instabilités actuelles, les mesures imaginées dans les instances internationales de supervision ne semblent pas vouloir/pouvoir aller au-delà de quelques retouches visant à calmer les ardeurs du moment et restent attachées à des positions antérieures, exprimées en 2006 par la Commission européenne. En effet, cette dernière précise que les solutions centralisées doivent être appliquées lorsqu'elles sont strictement nécessaires et que la régulation des établissements de crédit doit être principalement fondée sur leur évaluation par des agences de notation «bien gérées » et transparentes :
«À la demande du Parlement européen, la Commission a soigneusement examiné s'il y avait ou non lieu de prévoir de nouvelles propositions législatives en vue de réguler les activités des agences de notation. Sa conclusion est qu'aucune initiative législative nouvelle n'est nécessaire à l'heure actuelle. Un des principes fondamentaux du « mieux légiférer » est de veiller à ce que le recours aux solutions législatives ne soit envisagé que lorsque ces solutions sont strictement nécessaires à la réalisation d'objectifs des politiques publiques. La Commission est convaincue que la pertinence d'une nouvelle initiative législative dans ce domaine n'est toujours pas démontrée. (...) Dans son avis à la Commission, le CERVM a également indiqué qu'un équilibre approprié entre législation et autoréglementation avait été trouvé, et qu'il n'y avait donc pas lieu d'envisager d'autres initiatives législatives pour l'instant » (Journal officiel, 2006).

Bien que la Commission revienne sur cette question dans son règlement sur les agences de notation, publié dans le Journal officiel de l'Union européenne du 17 novembre 2009, elle maintient l'évaluation par les agences de notation privées comme principale ligne conductrice de la régulation bancaire et financière en leur demandant de résoudre, de façon appropriée, les éventuels conflits d'intérêt. De même, dans les orientations du Comité de Bâle sur les accords de troisième génération (Bâle III), annoncées en décembre 2010, bien qu'une définition plus stricte du capital des établissements à 
surveiller eut été adoptée et d'autres ratios ajoutés, la base de calcul pour la pondération des risques sur les actifs reste la même, fondée sur le principe d'IRB et de notations des agences privées (Lannoo, 2011) en laissant en suspens la question du conflit d'intérêt.

Pour éviter ces écueils récurrents dans les mécanismes de régulation en vigueur, il conviendrait d'éviter en même temps une confusion génétique que l'on rencontre souvent dans les débats entre l'ouverture des marchés et la déréglementation. L'ouverture est en général confondue avec la déréglementation. Or, une plus grande liberté des opérations privées, censée accroître la créativité et l'efficacité économiques, semble au contraire nécessiter plus de réglementation ciblée et en temps réel parce que les interdépendances entre les différents compartiments des économies s'accroissent et il ne devient plus possible de séparer, en cas de difficulté spécifique aux marchés financiers, les activités réelles des activités financières. Faire confiance mais surveiller et vérifier en permanence est pourtant un mot d'ordre qui avait été prononcé par les gouvernements économiquement libéraux depuis au moins le Président Ronald Reagan.

\section{Conclusion}

Il y a une constante dans les comportements des agents économiques sur les marchés qui, bien que rationnels sur le plan individuel, peuvent se révéler collectivement désastreux. Cette constante est annoncée par Kindleberger comme le comportement qui consiste pour chaque individu à agir comme un » fou » lorsque tout le monde agit follement ${ }^{30}$. Ainsi, Chuck Prince, alors président du Citigroup, avait annoncé en juillet 2007 que

«Lorsque la musique s'arrête, en termes de liquidité, les choses seront compliquées. Mais tant que la musique continue, vous devez rester debout et continuer à danser. Nous dansons encore et toujours $»^{31}$.

41 Cette forme de réaction des acteurs du marché traduit aussi la validité du principe de sophisme de composition qui, dans le cas précis, fait que l'agrégation des agents individuellement rationnels ne débouche pas trivialement sur une société rationnelle (Ülgen, 2011).

Bien que la tâche soit difficile comme le souligne A. Greenspan (2007) :

«(...) les bulles ne peuvent être désamorcées sans danger par une politique monétaire ou par d'autres initiatives avant que la fièvre spéculative ne s'éteigne d'elle-même. Il est clair que les banques centrales des différents pays auraient pu faire que très peu pour tempérer la manifestation la plus récente de cette disposition humaine à l'euphorie (...) »,

la recherche devrait porter sur les conditions cohérentes d'une restructuration des économies monétaires de marché que certains appelleraient la re-réglementation. Toutefois, ici, la re-réglementation ne signifie pas nécessairement un retour aux modes de régulation isolationnistes du passé, les pratiques du capitalisme étant depuis lors modifiées ; mais une régulation qui doit être repensée en termes des objectifs collectifs de développement stable aussi en phase avec les valeurs du libéralisme (pas nécessairement économique). Mais pour ce faire, il semble nécessaire de questionner certains principes fondamentaux du mode de régulation actuel des économies de marché avec une vision à plus long terme avant d'orienter la réflexion sur les réponses et d'établir des certitudes qui sont davantage préférées par les décideurs mais peu propices à l'assainissement des mécanismes économiques. 
43 éviter dans le futur. Par exemple, axées sur les taux d'inflation et le niveau des prix depuis plusieurs décennies, les banques centrales ne s'intéressent guère à l'évolution du crédit ni à celle du prix des actifs. Par conséquent, leurs interventions ne prennent que très peu en compte les hausses euphoriques des valorisations boursières et l'accroissement de l'endettement des diverses catégories d'agents. Prises au dépourvu en cas de crise, les Autorités adoptent alors des politiques expansionnistes qui deviennent le terreau pour une nouvelle bulle sans que les règles de fonctionnement des marchés ne soient révisées d'une façon cohérente. Une première vague d'aisance monétaire, face à de nombreuses faillites des établissements bancaires, est ensuite suivie par une deuxième vague de retour à des politiques économiques de rigueur face aux dettes publiques colossales, en hypothéquant les perspectives de reprise au profit d'une fidélité au conservatisme néolibéral (Ülgen, 2012).

P. Artus remarque ainsi que si rien n'est modifié dans le schéma d'intervention des banques centrales «les politiques monétaires deviendront inefficaces pour relancer le crédit et les économies, et la "dernière crise financière" se transformera en déflation où l'abondance de liquidité ne servira plus à rien" (2007, p. 230) en mettant les économies dans une trappe monétaire. En effet, les réactions des banques centrales aux crises par des politiques monétaires expansionnistes permettent, dans une certaine mesure, d'empêcher que la crise ne s'aggrave, mais elles participent aussi de l'alimentation des bulles en se focalisant exclusivement sur l'objectif du niveau des prix au lieu d'évaluer à chaque période l'orientation des autres grandeurs économiques. Il semble plus judicieux, par conséquent, de repenser les objectifs des politiques monétaires (sortir des limites des approches purement réelles) parallèlement à une conception internationalement coordonnée des mécanismes de régulation et de surveillance en considérant la stabilité des systèmes monétaires et financiers comme un bien public international.

Depuis le déclenchement de la crise en 2007, de nombreux travaux effectués proposent des modèles alternatifs en vue d'organiser des mécanismes de régulation financière plus pertinents tant dans leurs principes fondateurs que dans leurs modalités d'application (Brunnermeier et al. 2009) et en vue de modifier les orientations en matière des régimes d'accumulation dominants (Lapavitsas et al, 2010). Bien qu'il ne soit pas dans l'objectif de cet article de passer en revue ces différents travaux et de présenter des recommandations détaillées de politique économique et de régulation, il serait judicieux de remarquer que de nouvelles réflexions sur les principes et croyances qui orientent les mécanismes de fonctionnement des marchés et de régulation financière devraient être entreprises. Ces réflexions devraient viser l'analyse d'un certain nombre de principes afin de rendre compatibles les mécanismes de surveillance et d'intervention nationaux et internationaux autour des règles de coordination communes et proactives.

$\mathrm{Au}$ vu des résultats du présent travail, chercher à éviter l'écueil de la confusion entre l'économie de marché et l'absence de régulation collective se présente comme une première prérogative. L'évaluation des marchés et de l'état de l'endettement des différents acteurs doit se faire non seulement sur le plan micro-individuel mais aussi et surtout du point de vue macroéconomique, ce qui fait inéluctablement appel à une refonte des mécanismes de régulation dans une perspective macroéconomique. La régulation macroéconomique ne devrait pas être entendue comme un choix politique dont on pourrait se passer grâce aux modalités d'autorégulation que les marchés et les acteurs privés seraient en mesure de réinventer grâce à leur imagination innovante. La 
régulation macroéconomique est une condition sine qua non pour renforcer la stabilité systémique et élargir ainsi le domaine de viabilité de l'économie. Dans une économie de marché dont la dynamique dominante est fondée sur les décisions des acteurs privés et décentralisés, la viabilité du système n'est pas du ressort des marchés alors qu'elle est indispensable pour l'heureux exercice de l'initiative privée.

Dans cet objectif, deux questions sont à considérer. La première est de voir comment on pourrait éviter la confusion entre le processus d'innovation financière et les procédures de leur évaluation privée. À ce propos, il serait souhaitable de transformer les agences de notation en des sociétés d'audit, interdites d'intervenir comme conseillers auprès des établissements à évaluer. La deuxième est l'orientation des innovations financières. Remplacer les disponibilités de financement fondées sur les actifs financiers structurés par des formules de crédit à long terme, catégorisées en fonction de la nature des projets à financer (achats de biens finaux pour les ménages, besoins d'investissement pour les entreprises, etc.), permettrait en partie d'éviter que les opérations de spéculation et les opérations de financement à long terme ne se confondent et ne débouchent sur des prises de positions interdépendantes entre différents sphères et besoins à sensibilités distinctes dans l'économie. Dans cette lignée, De Grauwe (2008) avance l'idée de séparer les banques commerciales et les banques d'investissement, seules les premières devant bénéficier de l'intervention d'un prêteur en dernier ressort.

Il convient aussi de remarquer que dans la recherche d'une orientation alternative, les pays doivent agir de concert car les systèmes financiers ainsi que les économies nationales sont très interdépendants. Les capitaux peuvent se déplacer soudainement d'un marché à l'autre en créant des chocs d'envergure sur les économies et en accentuant les difficultés en termes de liquidité et/ou d'insolvabilité des établissements à effets systémiques. Lorsqu'une économie prend des mesures unilatérales, les asymétries entre les différentes régions se révèlent rapidement alors que la crise revêt un caractère global et n'épargnera aucune économie individuellement dans le cadre des interdépendances fortes comme c'est le cas aujourd'hui. Les mesures de régulation unilatérales sont aussi susceptibles de créer une course au moins-disant dans les réformes de régulation. Certaines alternatives, comme les plans de coordination internationale de Keynes de 1942-44 ou de Triffin de 1960, existent mais ils seraient politiquement irréalistes dans le contexte mondial actuel. Or, il semble aussi que «après une ou deux autres crises [de grande ampleur], l'impossible peut commencer à sembler réaliste » (Rogoff, 1999, p. 28). Dans une réforme éventuelle du système monétaire international, au-delà des principes actuellement dominants d'asymétrie et de subsidiarité qui ont manifestement échoué dans leurs objectifs, l'approfondissement des propositions des plans Keynes-Triffin à travers l'analyse Minskienne peut constituer une option sérieuse prometteuse.

Une refondation du Bretton Woods ne parait pas capable de constituer une alternative pertinente dans la mesure où l'évolution des marchés financiers, qui s'est révélée problématique, a été soutenue aussi par les institutions de coordination internationale de Bretton Woods, comme le FMI, dont les références politiques restent ancrées dans le libéralisme économique. Eichengreen (2008) souligne, à ce propos, la perte de crédibilité des programmes passés du FMI appliqués dans les pays émergents et propose la création d'une Organisation mondiale de la finance, proche du modèle de l'Organisation mondiale du commerce. Une question cruciale qui reste posée à ce jour est de savoir si l'asymétrie du système monétaire international, qui permet d'alimenter les déséquilibres aussi bien dans les économies du centre (États-Unis, Europe) que dans les économies périphériques 
(économies émergentes), pourrait être réduite à travers le régionalisme financier (Lombardi, 2010) dont les variantes actuellement imaginées se présentent sous la forme d'un fédéralisme conservateur limité dans l'Union européenne ou d'alternatives du type Initiative Chiang Mai pour les pays de l'Asie du Sud-Est et Banque du Sud pour les pays d'Amérique latine, qui cherchent à se détacher de l'attraction du Bretton Woods. Ces initiatives pourraient permettre de multi-polariser le Système monétaire international mais elles ne seront pas capables de produire de véritables solutions si elles s'érigent sur des principes asymétriques centre-périphérie.

Une nouvelle coordination/coopération monétaire et financière internationale doit être fondée sur de nouveaux principes symétriques entre les parties mais aussi sur l'objectif de la viabilité financière globale dépendant des règles macro-prudentielles, détachées de toute croyance aveugle en la capacité d'auto-ajustement des marchés décentralisés.

\section{BIBLIOGRAPHIE}

Aglietta, M. (1998), « La maîtrise du risque systémique international », Économie internationale, 76 (4), 41-67.

Aglietta, M. (2003), « Le risque systémique dans la finance libéralisée », Revue d'économie financière , 70, 33-50.

Aglietta, M. et Berrebi, L. (2007), Désordres dans le capitalisme mondial, Odile Jacob, Paris.

Artus, P. (2007), « Que devraient faire aujourd'hui les banques centrales ? », dans Regards croisés sur l'économie, $\mathrm{N}^{\circ}$ 3, La Découverte, 224-230.

Artus, P., Betbèze, J-P., de Boissieu, C., Capelle-Blancard, G. (2008), La crise des subprimes

Rapport du Conseil d'analyse économique, $\mathrm{N}^{\circ} 78$, La Documentation française.

Akerlof, G. A. (2005), Explorations in Pragmatic Economics, Selected Papers of G. A. Akerlof and CoAuthors, Oxford University Press.

Akerlof, G. A. et Shiller, R. J. (2009), Les esprits animaux. Comment les forces psychologiques mènent la finance et l'économie, Traduction française, Pearson Education France, Paris.

Barth, J. R., Caprio, G. and Levine, R. (2006), Rethinking Bank Regulation, Cambridge University Press.

Bhattacharya, S., Boot, W. A. and Thakor, A. V. (1998), "The Economics of Bank Regulation", Journal of Money, Credit, and Banking, 30(4), 745-770.

Bourghelle D., Brandouy O., Gillet R. et A. Orléan (éds.) (2005), Croyances, représentations collectives et conventions en finance, Paris, Economica.

Boyer, R. Dehove, M. et Plihon, D. (2004), Les crises financières, Rapport du Conseil d'analyse économique, $\mathrm{N}^{\circ} 50$, La Documentation française.

Brunnermeier, M. et al. (2009), The Fundamental Principles of Financial Regulation, Geneva Reports on the World Economy 11, International Center for Monetary and Banking Studies. 
Bryant, J. (1980), “A Model of Bank Reserves, Bank Runs and Deposit Insurance”, Journal of Banking and Finance, 4, 335-344.

Cafaggi, F. (2004), « Le rôle des acteurs privés dans le processus de régulation : participation, autorégulation et régulation privée ", Revue française d'administration publique, $\mathrm{N}^{\circ} 109,23-35$.

Cantor, R. and Mann, C. (2009), “Are Corporate Bond Ratings Procylical?: An Update”, Moody's Investor Services, Moody's Global Credit Policy, Special Comment, May.

Capie, F. and Wood, G. E. (eds.) (1986), Financial Crises and the World Banking System, Macmillan. Das, D. K. (ed.) (2003), An International Finance Reader, Routledge.

De Grauwe, P. (2008), "Returning to narrow banking”, in B. Eichengreen and R. Baldwin (eds.) What G20 leaders must do to stabilise our economy and fix the financial system, A VoxEU.org publication, CEPR, London, pp. 37-39.

Diamond, D. and Dybvig, P. (1983), “Bank Runs, Deposit Insurance, and Liquidity”, Journal of Political Economy, 91, 401-419.

Eichengreen, B. (2008), "Not a New Bretton Woods but a New Bretton Woods process", in B. Eichengreen and R. Baldwin (eds.) What G20 leaders must do to stabilise our economy and fix the financial system, A VoxEU.org publication, CEPR, London, pp. 25-27.

Fama, E. F. (1965), “The behaviour of stock-market prices”, The Journal of Business, 38(1), 34-105.

Fama, E. F. (1970), "Efficient capital markets: a review of theory and empirical work", The Journal of Finance, 25(2), 383-417.

Fama, E. F. (1980), "Banking in the theory of finance", Journal of Monetary Economics, 6, 39-57.

Festinger, L. (1957), A Theory of Cognitive Dissonance, Stanford University Press.

Goodhart, C.A.E. and Illing, G. (2002), Financial Crises, Contagion and the Lender of Last Resort: A Reader, UK: Oxford University Press.

Greenspan, A. (2007), “The Roots of the Mortgage Crisis”, The Wall Street Journal, 12 December 2007.

Honohan, P. and Laeven, L. (eds.) (2005), Systemic Financial Crises, Cambridge University Press. Hubbard, P. (ed.) (1991), Financial Markets and Financial Crises, Chicago University Press.

International Monetary Fund (2003), Global Financial Stability Report, September.

Journal officiel de l'Union européenne (2006), Communication de la Commission sur les agences de notation, (2006/C 59/02), 11.3.2006.

Journal officiel de l'Union européenne (2009), Le règlement $N^{\circ} 1060 / 2009$ sur les agences de notation de crédit, L302, 52 année, 17 novembre 2009.

Kaplan, S. and Stein, J. (1993), "The evolution of buyout pricing and financial structure in the 1980s", The Quarterly Journal of Economics, May, 313-357.

Kindleberger, C. P. and Laffargue, J. P. (eds.) (1982), Financial Crises. Theory, History and Policy, Cambridge University Press-Editions de la Maison des Sciences de l'Homme.

Kindleberger, C. P. (1989), Manias, Panics, and Crashes: A History of Financial Crises, $2^{\text {nd }}$ edition, Macmillan.

Kindleberger, C. P. (1997), “Financial Asset Inflation. Manias and How to Prevent Them”, Interview with Charles P. Kindleberger, Challenge, 40(6), pp. 21-31. 
Klein, L. R. and Shabbir, T. (eds.) (2006), Recent Financial Crises, Edward Elgar.

Lacoue-Labarthe, D. (1992), « Les crises financières et leur propagation internationale », dans E. Girardin (éd.), Finance internationale, Economica, 407-434.

Lannoo, K. (2011), “The forest of Basel III has too many trees”, Centre for European Policy Studies Commentary, 10 February 2011, http://www.ceps.eu.

Lapavitsas, C. et al. (2010), “Eurozone Crisis: Beggar Thyself and Thy Neighbour”, Research on Money and Finance Occasional Report, March, www.researchonmoneyandfinance.org

Lindbeck, A. (1979), “Comment on 'International Coordination of National Economic Policies' ”, in S. I. Katz (ed.), US-European Monetary Relations, American Enterprise Institute for Public Policy Research, Washington, D. C.

Llewellyn, J. (2008), “Anatomy of a Global Financial Crisis”, TheWorldToday.org, November, 64(11), 13-15.

Lombardi, D. (2010), “Financial Regionalism: A Review of the Issues”, The Brookings Institution Issues Paper, November.

Lorenzi, J.-H. (2008), « Les nouveaux acteurs de la finance », Regards croisés sur l'économie, $\mathrm{N}^{\circ}$ 3, La Découverte, 20-28.

Mayes, D. G. and Wood, G. E. (eds.) (2007), The Structure of Financial Regulation, Routledge.

Minsky, H. P. (1982), Can "It" Happen Again? Essays on Instability and Finance, Armonk, N.Y., Sharpe Inc.

Minsky, H. P. (1986), Stabilizing an Unstable Economy, Yale University Press.

Mishkin, F. S. (ed.) (2001), Prudential Supervision. What Works and What Doesn't, A National Bureau of Economic Research Conference Report, The University of Chicago Press.

Orléan, A. (2004), Analyse économique des conventions, $2^{\mathrm{e}}$ édition, PUF.

Report (2009), The High-Level Group on Financial Supervision in the EU, Chaired by Jacques de Larosière, Brussels, 25 February 2009.

Reinhart, C. M. and Rogoff, K. S. (2008), “This Time is Different: A Panoramic View of Eight Centuries of Financial Crises", manuscript, Harvard University, April 2008.

Rogoff, K. (1999), « International Institutions for Reducing Global Financial Instability », Journal of Economic Perspectives, 13(4), 21-42.

Portes, R. and Swoboda, A. K. (eds.) (1987), Threats to International Financial Stability, Cambridge University Press.

Securities and Exchange Commission (2003), "Report on the Role and Function of Credit Rating Agencies in the Operation of the Securities Markets," As required by Section 702(b) of the Sarbanes-Oxley Act of 2002, January.

Sgard, J. (2002), L'économie de la panique : faire face aux crises financières, Paris : La Découverte.

Shiller, R. J. (2005), Irrational Exuberance, 2nd Edition, Princeton, NJ, Princeton University Press.

Sy, A. N. R. (2009), The Systemic Regulation of Credit Rating Agencies and Rated Markets, IMF Working Paper, WP/09/129.

Ülgen, F. (1994), L'endogénéité de la monnaie : ébauche d'une théorie de l'économie monétaire, Thèse de doctorat, Université de Paris X - Nanterre. 
Ülgen, F. (2011), « Crise systémique et crise des fondements de la régulation financière », à paraître dans Économie appliquée, $\mathrm{N}^{\circ} 4$.

Ülgen, F. (2012), «La stabilité de l'espace monétaire européen et le rôle international de l'euro », à paraître dans $\mathrm{P}$. Bance (éd.), L'action publique dans la crise : vers un renouveau en France et en Europe ?, Presses Universitaires de Rouen et du Havre, PURH.

Wolfson, M. H. (1986), Financial Crises. Understanding the Postwar U.S. Experience, Armonk, N.Y., Sharpe Inc.

\section{NOTES}

3. Ces erreurs peuvent être définies comme les déséquilibres qui apparaissent suite à l'incompatibilité macroéconomique des décisions et actions microéconomiques.

4. Cet article n'a pas pour objectif de présenter ce paradigme mais de souligner qu'il a été le fondement conceptuel des orientations suivies dans la restructuration des marchés bancaires et financiers et dans l'élaboration des politiques de (dé)réglementation depuis les années 1970-80.

5. Dans une optique néoclassique de marchés parfaitement concurrentiels, les prix constituent la seule information nécessaire et suffisante que les agents peuvent et doivent disposer afin de prendre des décisions compatibles avec un équilibre global des marchés.

6. Il convient de remarquer ici que cette conception de l'efficience du mécanisme de prix de marchés libres est explicitement (et aussi inévitablement) fondée sur une vision a-monétaire de l'économie. L'affirmation de Fama est, on ne peut plus, claire: «Since the economy (...) is basically non-monetary, with some real good serving as numéraire, price level determinacy reduces to a standard problem concerning the existence of a stable equilibrium in a nonmonetary system », (1980, p. 44). Pour une discussion sur ce point, voir Ülgen (1994).

7. Je dois cette remarque à l'un des rapporteurs.

8. À titre d'exemple récent, on peut considérer le retrait, en 1999, de la loi Glass-Steagall de 1934 qui séparait les banques commerciales et les banques d'investissement aux États-Unis.

9. Parallèlement, la vague de mondialisation des années 1990 a mis non seulement la dette publique des économies développées mais aussi l'endettement des pays en développement -de plus en plus intégrés dans les circuits internationaux de financement- au cœur des marchés financiers, développés en produits et processus. Dans le cas des pays avancés, Aglietta et Berrebi (2007) soulignent que les déséquilibres nord-américains ne sont pas seulement dus au fonctionnement des marchés puisqu'ils sont rendus possibles aussi par le double déficit des ÉtatsUnis.

10. Par exemple, aux États-Unis avec les difficultés des Caisses d'épargne en 1987-88 et l'éclatement de la bulle des valeurs technologiques au début des années 2000.

11. Par exemple, en Asie avec les crises de 1997-98, en Argentine, au Brésil et en Turquie dans les années 1990 et 2000.

12. Les positions courtes -la valeur totale des produits dérivés vendus- et les positions longues la valeur totale des produits achetés- sont égales (mais, néanmoins, d'une maturité mal assortie). 13. Les différents fonds communs de créance ou de titrisation qui se financent par des émissions de titres négociables adossés aux actifs.

14. Par exemple, les papiers commerciaux adossés aux actifs.

15. IRB peut prendre la forme d'IRB-fondation, dans laquelle l'établissement estime sa probabilité de défaut de contrepartie et le taux de perte en cas de défaut sur la ligne de crédit est imposé par le régulateur national, ou la forme d'IRB-avancée, dans laquelle la banque est maîtresse de toutes les composantes de son indicateur. 
16. Ce ratio inclue les risques opérationnels sans réduire les valeurs de référence des ratios, $8 \%$ des fonds propres pour les engagements risqués et $4 \%$ pour les engagements totaux.

17. Il convient aussi de souligner que cette nouvelle réglementation est une recommandation dont l'application effective est laissée aux autorités nationales.

18. Ce qui est un problème de fond puisque la validation ex post de la pertinence d'une méthode d'évaluation n'a aucun sens face à la crise, qui est elle-même un résultat observé ex post de l'accroissement des « impertinences ».

19. Les crédits structurés sont établis sur le découpage des titres selon les différences qualitatives de leurs caractéristiques et sur leurs regroupements en tranches plus homogènes, les agences déterminant le taux d'intérêt associé à chaque tranche particulière. Toutefois, comme la notation est déterminée a priori au moment de l'élaboration des différentes tranches et non à partir de leur résultat final, l'établissement des catégories (les tranches) et leur notation s'effectue à travers la collaboration entre banques et agences de notation.

20. Un bien public est défini comme étant non rival (son utilisation simultanée en un nombre quelconque de fois par un nombre quelconque d'individus ne réduit en rien sa disponibilité et sa qualité), partiellement exclusif (partiellement appropriable puisque son usage par d'autres ne peut être contrôlé que partiellement par son détenteur) et ayant un faible coût de production et de transmission (son coût marginal tend vers zéro). L'évaluation concerne une activité et un produit qui auraient donc ces caractéristiques empêchant les mécanismes de prix concurrentiels d'en assurer la production optimale. De surcroît, la différence entre les avantages sociaux et les avantages privés (en faveur des premiers) de l'activité d'évaluation empêcherait que le niveau optimal de l'évaluation puisse être atteint par les seuls mécanismes de marché.

21. Je dois cette remarque à l'un des rapporteurs de la revue.

22. En effet, le marché de notation est dominé principalement par quelques grandes agences comme Fitch, Moody's et Standard and Poor's.

23. La succession «folle» des menaces de dégradation des notations sur les dettes souveraines, de complication des difficultés d'assainissement économique et financière dans l'Union européenne et des annonces sans retenu des politiques d'ajustement et d'austérité traduit le degré de confusion intellectuelle, économique et politique dans laquelle se trouvent aujourd'hui à la fois les décideurs politiques, les économistes conseillers et les citoyens.

24. Le cas de l'Union européenne constitue un bon exemple dans ce sens dans la mesure où l'existence d'une banque centrale européenne supranationale ne répond ni dans ses statuts ni dans ses attributs à la condition d'une coordination unifiée inter-nations à l'intérieur de l'espace européen, les différents pays suivant, selon le principe de subsidiarité, différents mécanismes de surveillance de leurs marchés monétaires et financiers. Mais devant l'ampleur de la crise actuelle et à condition d'être effectivement opérationnels - au grand dam des autorités britanniques et de la City -, les conclusions du Rapport Larosière de février 2009 et les nouveaux mécanismes de coordination de la supervision en Europe (comme le Conseil européen du risque systémique (CERS) et le Système européen de surveillance financière (SESF)) semblent aller dans le sens d'un renforcement des modalités de supervision et d'intervention sur les marchés monétaires et financiers.

25. Sauf si on les suppose omniscients et atemporels comme dans un modèle d'équilibre général avec marchés complets.

26. Ce qui est bien le mécanisme d'arbitrage des marchés qui devrait, selon les modèles d'efficience, équilibrer les offres et les demandes.

27. Il peut s'agir des risques de crédit sur les engagements de dette et de contreparties et des risques de marché sur les prix d'actifs et la liquidité des acteurs engagés.

28. Le système productif se développant et permettant d'améliorer le niveau des revenus dans l'ensemble de l'économie et alimentant ainsi des anticipations de plus en plus optimistes sur l'avenir et réduisant la vigilance des agents quant à la qualité des projets à financer. 
29. Il s'agit des mécanismes législatifs et économiques qui offriraient les bonnes incitations aux acteurs privés.

30. Ce qui avait été souligné aussi par Keynes dans la Théorie générale (1936) à travers l'exemple de concours de beauté et étudié plus récemment par Akerlof et Shiller dans leur ouvrage de 2009. 31. Cité dans Llewellyn (2008), traduction personnelle.

\section{RÉSUMÉS}

Les modèles d'évaluation interne décentralisée (autorégulation), qui se substituent aux mécanismes de régulation publique, ne sont pas en mesure d'avoir une vision macroéconomique à long terme ni de prendre en compte les interdépendances entre les acteurs privés et les marchés. Dès lors, ils se révèlent incapables de faire face à des situations de crise systémique. De surcroît, les schémas de supervision libérale réduisent le champ d'action des Autorités monétaires et limitent la portée systémique de leur intervention en cas de crise d'envergure. Par conséquent, l'absence de mécanismes de régulation macroéconomique apparaît comme l'une des raisons de l'apparition et la persistance des crises financières généralisées. Une relecture du schéma Minskien d'instabilité financière et des résultats des modèles de conventions, de financement par LBO et de dissonance cognitive montre que la crise financière actuelle remet en question deux principes du mode de régulation financière du capitalisme moderne: 1) La croyance en la capacité des marchés à corriger les erreurs de jugement des acteurs décentralisés sans une intervention publique structurelle ;2) L'efficacité d'une autorégulation des marchés par rapport aux schémas de régulation publique. Ces principes se révèlent incapables aujourd'hui d'assurer la continuité des rapports économiques sous leur forme actuelle si bien qu'un débat de fond sur de nouveaux mécanismes de régulation macro-prudentielle, visant à renforcer la viabilité des systèmes monétaires et financiers, paraît nécessaire.

Decentralized internal rating based models (self-regulation) which are substituted to public regulation are not able to hold a long-term macroeconomic vision or to take into account interdependencies among private units and markets. Therefore, they seem to be unable to tackle with systemic crises. Moreover, liberal supervision schemes reduce the field of action of monetary authorities and limit the systemic range of their interventions in case of large crisis. Then the absence of macro-regulatory schemes reveals to be one of the causes of the appearance and the persistency of generalized financial crises. A reappraisal of the Minskian financial instability hypothesis and the results of models of conventions, of financing by LBO and of cognitive dissonance points out that the current financial crisis casts doubt on two principles of the way of regulation of modern capitalism: 1) The capacity of market mechanisms for correcting errors of judgment of decentralized actors without structural public interventions; 2) The efficiency of the self-regulation of markets regarding public regulation schemes. These principles turn out to be unable to ensure the continuity in market relations under their present form. So, new research becomes compulsory in order to imagine new macro-prudential mechanisms seeking to strengthen the viability of economic and monetary relations. 
INDEX

Code JEL G01 - Financial Crises, G18 - Government Policy and Regulation, P11 - Planning; Coordination; and Reform

Keywords : regulatory mechanisms, banking and financial crisis, viability

Mots-clés : mode de régulation, crise bancaire et financière, viabilité

\section{AUTEUR}

\section{FARUK ÜLGEN}

Maître de Conférences - Habilité à Diriger des Recherches ; Centre de Recherche en Economie de Grenoble (CREG) ; Faculté d'Economie de Grenoble - Université Pierre Mendès France - Grenoble 2 ; 1221, rue des résidences BP 4738040 Grenoble Cedex 09 - France ; Tél : +33(0) 476825458 (poste 84 60)- Fax : +33(0) 476825995 ; Ulgen.Faruk[at]upmf-grenoble.fr 